\title{
Prognostic significance of IL-17 mRNA expression in peritoneal lavage in gastric cancer patients who underwent curative resection
}

\author{
TAKESHI IIDA ${ }^{1}$, MAKOTO IWAHASHI $^{1}$, MASAHIRO KATSUDA $^{1}$, KOICHIRO ISHIDA $^{1}$, \\ MIKIHITO NAKAMORI ${ }^{1}$, MASAKI NAKAMURA ${ }^{1}$, TEIJI NAKA ${ }^{1}$, TOSHIYASU OJIMA ${ }^{1}$, \\ KENTARO UEDA $^{1}$, KEIJI HAYATA ${ }^{1}$, HIRONAO YASUOKA ${ }^{2}$ and HIROKI YAMAUE ${ }^{1}$ \\ ${ }^{1}$ Second Department of Surgery, School of Medicine, and ${ }^{2}$ Division of Pathology, Department of Clinical \\ Laboratory Medicine, School of Medicine, Wakayama Medical University, Wakayama 641-8510, Japan
}

Received October 23, 2013; Accepted November 11, 2013

DOI: $10.3892 / o r .2013 .2911$

\begin{abstract}
Peritoneal dissemination is frequently detected in patients with advanced gastric cancer. The peritoneal cavity is a compartment in which an immunologic host-tumor interaction can occur. There are no reports on the relationship between IL-17 expression in peritoneal lavage and prognosis in gastric cancer patients. Therefore, we investigated the expression of IL-17 mRNA in peritoneal lavage from gastric cancer patients and assessed the association of its expression with clinicopathological parameters and prognosis. Peritoneal lavage was obtained from 114 patients with gastric cancer at initial surgery. Seventy-nine patients underwent curative resection. Among these 79 patients, IL-17 mRNA expression was associated with the depth of tumor invasion $(\mathrm{P}<0.05)$. Twelve of the 79 patients who underwent curative resection died, and 9 of those 12 developed peritoneal metastasis. Notably, among the 79 patients who underwent curative resection, those with high expression of IL-17 mRNA in peritoneal lavage had significantly prolonged survival when compared to these patients with low expression of IL-17 mRNA in peritoneal lavage $(\mathrm{P}<0.05)$ as evidence by the survival curves. In a multivariate analysis, low expression of IL-17 mRNA in peritoneal lavage and tumor size were found to be independent significant predictive factors for prognosis (HR, 7.91; 95\% CI, 1.65-38.03) in the patients who underwent curative resection. IL-17 mRNA expression in peritoneal lavage is a reliable prognostic factor for patients undergoing curative resection for gastric cancer. Low IL-17 expression in the peritoneal cavity may correlate with cancer development in the peritoneal cavity in patients with gastric cancer.
\end{abstract}

Correspondence to: Dr Makoto Iwahashi, Second Department of Surgery, School of Medicine, Wakayama Medical University, 811-1 Kimiidera, Wakayama 641-8510, Japan

E-mail: makoto@wakayama-med.ac.jp

Key words: gastric cancer, interleukin-17, peritoneal lavage, prognostic factor

\section{Introduction}

Although the prognosis of gastric cancer has improved with the development of early diagnosis and new therapeutic strategies, it remains one of the main causes of cancer-related mortality worldwide (1), with peritoneal carcinomatosis, often associated with malignant ascites, being the most frequent cause of death in patients with advanced gastric cancer. Peritoneal dissemination is considered to arise from free cancer cells in the peritoneal cavity exfoliated from the serosal surface of the stomach penetrated by the primary tumor (2). Therefore, cytologic examination of peritoneal washes has been performed at laparotomy to detect free cancer cells in patients with advanced gastric cancer, and it is recognized as one of the most important prognostic factors (3-7). Since 1999, it has been reported that the presence of free cancer cells in the peritoneal cavity should be considered as an independent prognostic factor in patients with gastric cancer by the Japanese Gastric Cancer Association. Moreover, in the International Union Against Cancer's TNM classification 7th edition for gastric cancer, positive peritoneal cytology is defined as stage IV.

Therefore, detection of free cancer cells in peritoneal washes is a standard method for the assessment of risk for peritoneal carcinomatosis. However, patients who are diagnosed as having no free cancer cells in the peritoneal cavity by conventional cytology sometimes develop peritoneal recurrence after curative resection. This may occur since cytology is considered to have low sensitivity. In fact, recently, real-time RT-PCR techniques have been developed in order to increase the sensitivity of conventional peritoneal lavage cy tology $(8,9)$. Concerning another cause for the development of recurrent peritoneal disease, the cytokine network may play an important role in the immunosuppressive and immunostimulatory properties of cancer-related ascites fluid $(10,11)$. However, the role of cytokines produced by cells in the peritoneal cavity on tumor growth in gastric cancer patients is still unclear.

Th17 cells have recently been identified as having a distinct Th cell lineage and were found in an experimental animal model of cancer and in human cancers. Th17 cells have been found in several types of human cancers, such as ovarian, prostate, colorectal, and other malignancies, as well as gastric 
cancer (12-17). Research has found that IL-17 promotes tumor growth through angiogenesis and inflammation (18), and several other studies have demonstrated that IL-17 contributes to reduced tumor growth by promoting dendritic cell, cytotoxic $\mathrm{T}$ cell (CTL), and NK cell trafficking to, and retention within, the tumor microenvironment $(19,20)$. Therefore, whether these cells promote tumor growth or regulate antitumor responses remains controversial. In the case of human gastric cancer, there are several reports concerning the prevalence of Th17 cells in the tumor microenvironment, tumor-draining lymph nodes and peripheral blood $(12,13)$. In our previous study, the expression level of IL-17 mRNA in gastric tumors was associated with the depth of tumor invasion, lymphovascular invasion and lymph node involvement suggesting that IL-17 is clearly associated with tumor progression (21). However, since no previous studies have been carried out concerning the proportion of IL-17 in the peritoneal cavity in human gastric cancer patients, we hypothesized that the expression level of IL-17 mRNA in peritoneal lavage may be involved in the development of peritoneal carcinomatosis in gastric cancer.

In the present study, we quantitatively investigated expression of IL-17 messenger RNA (mRNA) in the peritoneal lavage of gastric cancer patients who underwent curative resection. The association of IL-17 expression levels with clinicopathological factors and prognosis was also assessed. Since IL-2 and IL-12 have been used for intraperitoneal immunotherapy in patients with various types of cancers, including gastric cancer (22-25), the possibility of IL-17 as a therapeutic target for patients with gastric cancer was also investigated.

\section{Materials and methods}

Patients. Included in the present study was a series of 114 patients (80 men and 34 women) with gastric cancer who underwent surgery at Wakayama Medical University Hospital (WMUH) from 2003 to 2006. At the beginning of the operation, we examined tumor metastases in the abdominal cavity. When it was thought that curative resection was possible, we performed gastrectomy with lymphadenectomy. We performed gastrectomy in 114 patients with gastric cancer. Seventy-nine patients underwent surgically curative resection and 35 underwent non-curative resection. None of the patients received anticancer therapy prior to surgery. Individuals with autoimmune disease, inflammatory bowel disease or viral infections were excluded. The clinicopathological characteristics of the 114 patients are summarized in Table IA. Clinical stages of the tumors were determined according to the International Union Against Cancer's TNM classification for gastric cancer. After surgery, all patients underwent a follow-up, with the median follow-up at analysis being 61 months (range, 1.3-98.5) for all patients. Every 3 to 6 months, physical examination, blood chemistry, including carcinoembryonic antigen (CEA) and cancer antigen (CA) 19-9, and computed tomography were performed for each patients. Written informed consent was obtained from all patients before participation in the present study. In addition, the local ethics committee of WMUH approved the study.

Preoperative peritoneal wash examination. At the beginning of each operation, $100 \mathrm{ml}$ saline was introduced into the
Douglas cavity and aspirated by gentle stirring. These washes were centrifuged at 1,800 rpm for $5 \mathrm{~min}$ to collect intact cells. A part of each peritoneal wash was examined cytopathologically after conventional Papanicolaou staining.

RNA extraction and DNA synthesis. Total RNA was extracted using an RNeasy Mini kit (Qiagen, Hilden, Germany) followed by RNase-Free DNase Set treatment (Qiagen). Complementary DNA was synthesized from $1 \mu \mathrm{g}$ of total RNA using a reverse transcription system (Promega, Madison, WI, USA) according to the manufacturer's instructions. Samples were stored at $-80^{\circ} \mathrm{C}$ until use.

Quantitative real-time RT-PCR. Quantitative real-time reverse transcription-polymerase chain reaction (RT-PCR) was performed with isolated total RNA $(1 \mu \mathrm{g})$ on the LightCycler system (Roche Molecular Biochemicals, Mannheim, Germany). The following oligonucleotide primers and hybridization probes were used: human IL-17 (GenBank accession no. NM 002190; 53-231 bp): sense, 5'-CTGGGAAGACCTCA TTGG-3'; antisense, 5'-CCTTTTGGGATTGGTATTGG-3'; fluorescein-labeled probe, 5'-TCCTCAGAATTTGGGC ATCCTGGATTTC-3'; and LC Red 640-labeled probe, 5'-TGGGATTGTGATTCCTGCCTTCACTATGG-3'; human glyceraldehyde 3-phosphate dehydrogenase (GAPDH; GenBank accession no. NM 002046; 746-1052 bp): sense, 5'-TGAACGGGAAGCTCACTGG-3'; antisense, 5'-TCC ACCACCCTGTTGCTGTA-3'; fluorescein-labeled probe, 5'-TCAACAGCGACACCCACTCCT-3'; and LC Red 640-labeled probe, 5'-CACCTTTGACGCTGGGGCT-3'. Primers and probes were designed by Nihon Gene Research Laboratories, Inc. (Miyagi, Japan). After $10 \mathrm{~min}$ of initial denaturation at $95^{\circ} \mathrm{C}$, the cycling protocol entailed 40 cycles of denaturation at $95^{\circ} \mathrm{C}(10 \mathrm{sec})$, annealing at $62^{\circ} \mathrm{C}(15 \mathrm{sec})$ and elongation at $72^{\circ} \mathrm{C}(8 \mathrm{sec})$. For GAPDH, the thermocycling protocol was the same, except that annealing was performed at $55^{\circ} \mathrm{C}(15 \mathrm{sec})$ and 50 cycles were run. On each run, all samples were quantified according to the LightCycler software program, version 3.8 (Roche Molecular Biochemicals). The levels of mRNA for IL-17 were corrected with GAPDH housekeeping control amplifications. We used the following for quantitative RT-PCR analysis: IL-17 ratio = IL-17 value/ GAPDH value $\mathrm{x} 10^{4}$.

Determination of the cut-off value. The cut-off value of the IL-17 mRNA ratio was determined as the median value based on the quantified values of 114 samples in the present study. The cut-off value was 1.22 .

Immunohistochemistry and quantitative microscopy. Sections $(4 \mu \mathrm{m})$ were prepared from paraffin-embedded blocks derived from gastric tumors. Sections were deparaffinized in xylene and graded alcohols, and rinsed in phosphate-buffered saline. Antigen retrieval from the tissues was carried out by autoclaving the tissues in $0.01 \mathrm{M}$ citrate buffer $\left(\mathrm{pH} \mathrm{6.0)}\right.$ at $100^{\circ} \mathrm{C}$ for $10 \mathrm{~min}$. The antibody used was goat anti-IL-17 (dilution at $10 \mu \mathrm{g} / \mathrm{ml}$; R\&D Systems, Minneapolis, MN, USA). The antibodies were incubated overnight at $4^{\circ} \mathrm{C}$. The immunocomplex was visualized by a polymer envision method, EnVision ${ }^{\mathrm{TM}}+$ Kit (DakoCytomation, Glostrup, Denmark). For quantification 

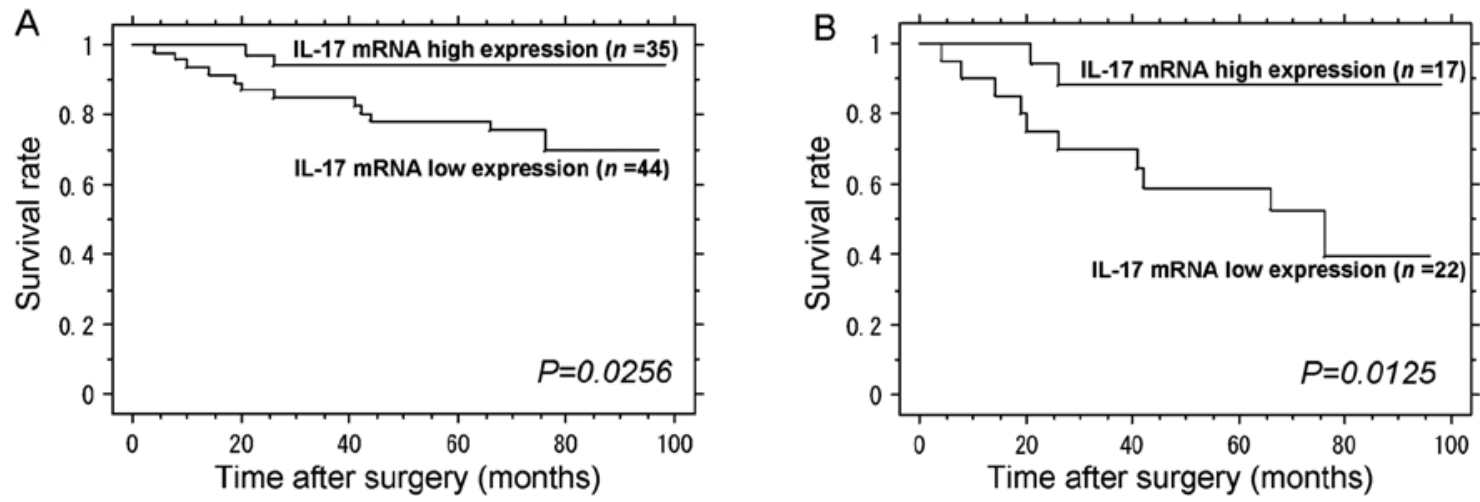

Figure 1. (A) Overall survival curves of the 79 gastric cancer patients who underwent curative R0 resection stratified according to IL-17 mRNA high or low expression in peritoneal lavage as determined by real-time RT-PCR. A significant difference in survival was noted between the IL-17 mRNA high expression and IL-17 mRNA low expression groups ( $\mathrm{P}=0.0256$; log-rank test). (B) The overall survival of the 79 patients who underwent curative resection with clinical stage II/III tumors according to IL-17 mRNA high or low expression in peritoneal lavage as determined by real-time RT-PCR. The IL-17 mRNA low expression group had a significantly poorer prognosis than the high expression group among the clinical stage II/III patients $(\mathrm{P}=0.0125, \log$-rank test).

of IL-17-positive cells, highly positive areas were initially identified by scanning tumor sections using light microscopy. Data were obtained by manually counting positively stained cells in five separate areas of intratumoral regions under $x 400$ high power magnification. The regions were counted by a pathologist who had no knowledge of the other clinicopathological features and survival outcomes.

Flow cytometry. For intracellular molecule measurements, cells were stimulated with PMA $(10 \mathrm{ng} / \mathrm{ml})$ and ionomycin $(500 \mathrm{ng} / \mathrm{ml})$ (Sigma-Aldrich Chemie GmbH, Steinheim, Germany) for $5 \mathrm{~h}$ in the presence of GolgiPlug (BD Biosciences, San Diego, CA, USA). Then, the cells were harvested and stained with PerCP-Cy5.5-conjugated anti-CD3 mAb, FITCconjugated anti-CD4 mAb, FITC-conjugated anti-CD8 mAb and FITC-conjugated anti- $\gamma \delta$ TCR mAb (BD Biosciences) for $30 \mathrm{~min}$ on ice. For intracellular staining, after fixation and permeabilization using BD Cytofix/Cytoperm (BD Biosciences), the cells were stained with PE-conjugated antiIL-17 mAb (BD Biosciences) for $30 \mathrm{~min}$ at $4^{\circ} \mathrm{C}$. After washing, the cells were analyzed by FACSCalibur, using CellQuest (BD Biosciences).

Statistical analysis. The Mann-Whitney test and the KruskalWallis test were used to determine statistical significance between the covariates. The Cox proportional hazards model was used to compute the univariate and multivariate hazards ratios for the study parameters. Survival curves were computed using the Kaplan-Meier method and compared by means of the log-rank test. The survival curve was calculated from the date of surgery. In Fig. 3, we used the Spearman rank correlation coefficient. All statistical analyses were performed with StatView 6.0 (Abacus Concepts, Inc., Berkeley, CA, USA) statistical software program. A value of $\mathrm{P}<0.05$ was considered to indicate a statistically significant result.

\section{Results}

Relationship between IL-17 mRNA and clinicopathological factors. To evaluate the biological significance of IL-17 expression in peritoneal lavage from patients with gastric cancer, the association between mRNA expression levels of IL-17 and clinicopathological factors was investigated. In all patients, the IL-17 mRNA expression level in the peritoneal lavage increased according to the depth of tumor invasion and peritoneal metastases, while the expression level was not associated with cytologic examination (Table IA). In the patients who underwent curative resection, IL-17 mRNA expression levels in peritoneal lavage increased according to the depth of tumor invasion (Table IB). On the other hand, no significant association was recognized between the expression level of IL-17 mRNA and histological type, lymph node metastases, lymphatic invasion, vessel invasion, clinical stage or tumor size.

Correlation between patient survival and IL-17 mRNA expression in peritoneal lavage. Kaplan-Meier survival curves indicated the overall survival of gastric carcinoma patients stratified according to the results of the IL-17 mRNA expression status in peritoneal lavage. The survival curves of all 114 patients displayed no significant difference between the IL-17 mRNA low expression group and the IL-17 mRNA high expression group (data were not shown). Importantly, however, based on the survival curves, among the 79 patients who underwent curative R0 resection, the patients in the IL-17 mRNA low expression group $(n=44)$ had a significantly poorer prognosis when compared with the patients in the IL-17 mRNA high expression group $(\mathrm{n}=35)$ (Fig. $1 \mathrm{~A} ; \mathrm{P}<0.05)$. During the median 61 months of postoperative surveillance, 14 (17.8\%) of the 79 patients who underwent curative resection died, and 12 (85.7\%) of these 14 patients developed peritoneal metastasis. Regarding peritoneal recurrence, 10 (22.7\%) of the 44 cases in the IL-17 mRNA low expression group developed peritoneal metastases, while $2(5.7 \%)$ of the 35 cases in the IL-17 mRNA high expression group developed peritoneal metastases.

Correlation between survival and IL-17 mRNA in peritoneal lavage in advanced gastric cancer. Among the patients who underwent curative resection with clinical stage II/III tumors, the prognosis of the IL-17 mRNA low group was significantly 
Table I. Clinicopathological data and IL-17 mRNA expression of the 114 patients and the 79 patients who underwent curative resection.

A, Data of the 114 patients

\begin{tabular}{lcll}
\hline & $\begin{array}{c}\text { No. of } \\
\text { patients }\end{array}$ & $\begin{array}{c}\text { Expression of } \\
\text { Factor }\end{array}$ & IL-17 mRNA \\
\hline
\end{tabular}

Age (years)

$\leq 65$

$>65$

0.448

Gender

Male

Female

Depth of tumor invasion

T1

$\mathrm{T} 2$

T3

T4

Lymph node metastasis

N0
N2
N3

Histological type

Differentiated

Undifferentiated

Lymphatic invasion

Negative
Positive
Vessel invasion
Negative
Positive

Peritoneal metastasis

Negative

Positive

Cytologic examination

Negative

Positive

$\begin{array}{ll}50 & 2.11 \pm 0.648 \\ 64 & 1.57 \pm 0.399 \\ & \\ 80 & 1.75 \pm 0.415 \\ 34 & 1.93 \pm 0.724 \\ & \\ 30 & 1.00 \pm 0.523 \\ 13 & 1.49 \pm 0.130 \\ 31 & 2.44 \pm 0.809 \\ 40 & 2.51 \pm 0.582\end{array}$

$43 \quad 2.25 \pm 0.681$

0.477

$28 \quad 1.26 \pm 0.581$

$21 \quad 1.61 \pm 0.708$

$22 \quad 1.84 \pm 0.894$

$\begin{array}{ll}54 & 2.08 \pm 0.601 \\ 60 & 1.55 \pm 0.423\end{array}$

0.387

$60 \quad 1.55 \pm 0.423$

$37 \quad 1.90 \pm 0.659$

0.338

$77 \quad 1.76 \pm 0.432$

$57 \quad 1.83 \pm 0.544$

$57 \quad 1.78 \pm 0.479$

0.292

$100 \quad 2.07 \pm 0.439$

$14 \quad 7.21 \pm 0.290$

$0.031^{\mathrm{d}}$

$91 \quad 1.22 \pm 0.591$

$23 \quad 1.89 \pm 0.404$

Stage $^{b}$

I

II

$40 \quad 1.95 \pm 0.660$

$18 \quad 2.29 \pm 0.109$

$21 \quad 1.38 \pm 0.784$

III

$33 \quad 1.64 \pm 0.531$

Tumor size $(\mathrm{cm})$

\begin{tabular}{llll}
$\leq 5$ & 60 & $1.75 \pm 0.516$ & 0.059 \\
$>5$ & 54 & $1.87 \pm 0.506$ & \\
\hline
\end{tabular}

poorer than that of the patients in the IL-17 mRNA high group (Fig. 1B; $\mathrm{P}<0.05$ ). From the point of view of the depth of the invasion, patients in the IL-17 mRNA low group had significantly poorer outcome than those in the IL-17 mRNA high
Table I. Continued.

B, Data of the 79 patients who underwent curative resection

\begin{tabular}{lccc}
\hline & $\begin{array}{c}\text { No. of } \\
\text { patients }\end{array}$ & $\begin{array}{c}\text { Expression of } \\
\text { IL-17 mRNA }\end{array}$ & P-value \\
\hline
\end{tabular}

Age (years)

$\begin{array}{llll}\leq 65 & 35 & 2.01 \pm 0.781 & 0.329\end{array}$

$>65 \quad 44 \quad 1.72 \pm 0.527$

Gender

Male

Female

$58 \quad 1.80 \pm 0.517$

0.975

$21 \quad 1.93 \pm 0.724$

Depth of tumor invasion

T1

$30 \quad 1.00 \pm 0.523$

$0.020^{\mathrm{c}}$

$\mathrm{T} 2$

$13 \quad 1.49 \pm 0.130$

$23 \quad 2.39 \pm 0.927$

T3

$13 \quad 2.90 \pm 0.124$

Lymph node metastasis

$\begin{array}{lcc}\text { N0 } & 43 & 2.25 \pm 0.681 \\ \mathrm{~N} 1 & 20 & 1.48 \pm 0.700 \\ \mathrm{~N} 2 & 10 & 1.90 \pm 0.106 \\ \mathrm{~N} 3 & 6 & 3.26 \pm 0.284\end{array}$

Histological type

Differentiated

41

$2.31 \pm 0.719$

0.613

Undifferentiated

38

$1.33 \pm 0.524$

Lymphatic invasion

Negative

34

$1.72 \pm 0.690$

0.118

Positive

$45 \quad 1.95 \pm 0.610$

Vessel invasion

Negative

$49 \quad 1.72 \pm 0.597$

0.087

Positive

30

$2.05 \pm 0.708$

Stage $^{b}$

I

$40 \quad 1.95 \pm 0.660$

0.497

II

$18 \quad 2.29 \pm 0.109$

$21 \quad 1.44 \pm 0.819$

Tumor size $(\mathrm{cm})$

$\begin{array}{lll}\leq 5 & 54 & 1.62 \pm 0.509 \\ >5 & 25 & 2.36 \pm 0.943\end{array}$

0.097

${ }^{\mathrm{a}}$ Expression of mRNA for IL-17 was corrected with GAPDH housekeeping control amplifications. Values represent mean \pm SEM. ${ }^{b}$ Stage according to the TNM classification for gastric cancer (UICC). ${ }^{\mathrm{c}} \mathrm{P}$-value of Kruskal-Wallis test as appropriate. ${ }^{\mathrm{d}} \mathrm{P}$-value of MannWhitney test as appropriate.

group for patients in the T2/3/4 subgroups (Fig. 2A; $\mathrm{P}<0.05$ ). In the $\mathrm{T} 4$ subgroup, patients with IL-17 mRNA low expression in peritoneal lavage had a significantly poorer survival than those with IL-17 mRNA high expression (Fig. 2B; P<0.05).

Preoperative peritoneal wash assay as an independent prognostic factor. We evaluated prognostic factors in the 79 patients who underwent curative $\mathrm{R} 0$ resection. With the overall 

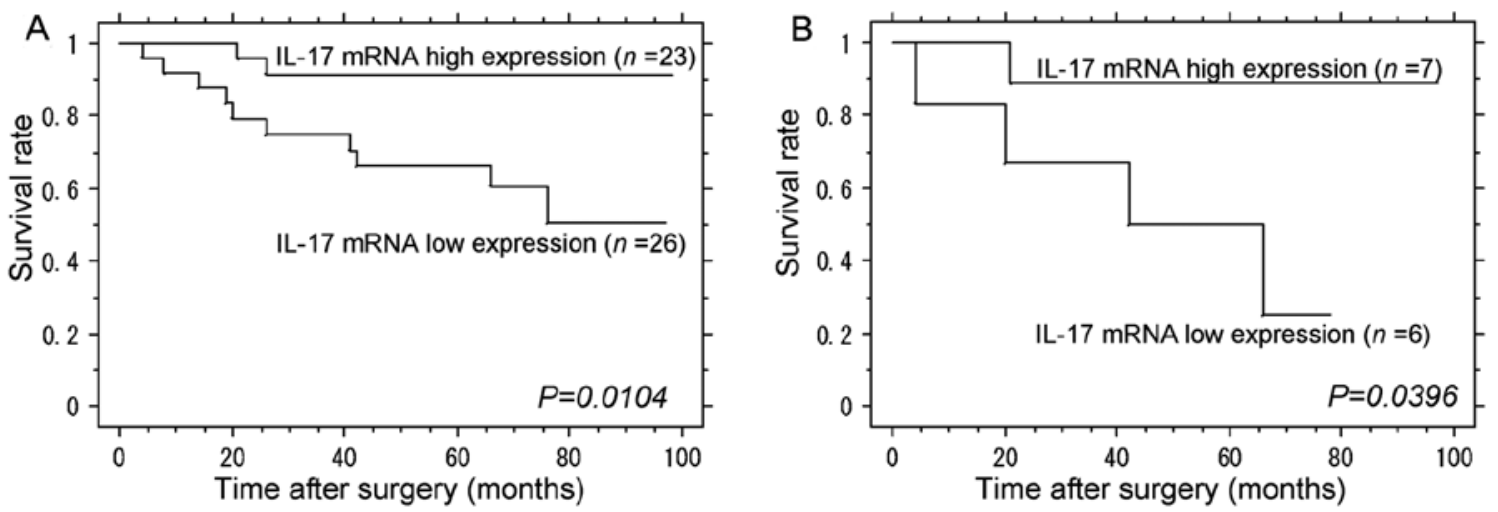

Figure 2. Overall survival curves of the 79 gastric cancer patients who underwent curative resection with pT2/3/4 stage tumors stratified according to IL-17 mRNA high or low expression in peritoneal lavage. (A) Overall survival of the 79 patients subdivided according to IL-17 mRNA high or low expression in peritoneal lavage with pT2/3/4 stage tumors. The IL-17 mRNA low expression group had a significantly poorer prognosis than the high expression group in the combined pT2/3/4 subgroup ( $\mathrm{P}=0.0104$; log-rank test). (B) Overall survival of the 79 patients with pT4 stage tumors subdivided according to IL-17 mRNA high or low expression in peritoneal lavage. The IL-17 mRNA low expression group had a significantly poorer prognosis than the high expression group among the pT4 patients $(\mathrm{P}=0.0396$; log-rank test $)$.

Table II. Univariate and multivariate analysis of the overall survival for the 79 patients who underwent R0 curative resection.

\begin{tabular}{|c|c|c|c|c|c|c|}
\hline \multirow[b]{2}{*}{ Variables } & \multicolumn{3}{|c|}{ Univariate analysis } & \multicolumn{3}{|c|}{ Multivariate analysis } \\
\hline & Hazard ratio & $95 \% \mathrm{CI}$ & P-value & Hazard ratio & $95 \% \mathrm{CI}$ & P-value \\
\hline \multicolumn{7}{|l|}{ Age (years) } \\
\hline$\leq 65$ vs. $>65$ & 1.62 & $0.542-4.840$ & 0.388 & - & - & - \\
\hline \multicolumn{7}{|l|}{ Gender } \\
\hline Male vs. female & 1.07 & $0.335-3.438$ & 0.906 & - & - & - \\
\hline \multicolumn{7}{|l|}{ Lymph node metastasis } \\
\hline Negative vs. positive & 9.98 & $1.296-76.95$ & 0.027 & 2.94 & $0.175-49.39$ & 0.454 \\
\hline \multicolumn{7}{|l|}{ Serosal invasion } \\
\hline Negative vs. positive & 9.04 & $2.014-40.53$ & 0.004 & 1.56 & $0.215-11.35$ & 0.659 \\
\hline \multicolumn{7}{|l|}{ Lymphatic invasion } \\
\hline Negative vs. positive & 9.98 & $1.296-76.95$ & 0.027 & 1.05 & $0.046-23.79$ & 0.976 \\
\hline \multicolumn{7}{|l|}{ Vessel invasion } \\
\hline Negative vs. positive & 10.38 & $2.320-46.42$ & 0.0022 & 4.16 & $0.459-37.68$ & 0.205 \\
\hline \multicolumn{7}{|l|}{ Histological type } \\
\hline Differentiated vs. undifferentiated & 2.16 & $0.720-6.497$ & 0.169 & - & - & - \\
\hline \multicolumn{7}{|l|}{ Tumor size $(\mathrm{cm})$} \\
\hline$\leq 5$ vs. $>5$ & 7.32 & $2.289-23.40$ & 0.0008 & 4.61 & $1.19-17.78$ & 0.027 \\
\hline \multicolumn{7}{|l|}{ IL-17 mRNA expression } \\
\hline Low expression vs. high expression & 4.69 & $1.049-20.99$ & 0.043 & 7.91 & $1.65-38.03$ & 0.0098 \\
\hline
\end{tabular}

CI, confidence interval.

survival as an endpoint, lymph node metastasis, serosal invasion, lymphatic invasion, vessel invasion, tumor size and IL-17 mRNA expression were found to be significant as prognostic factors by univariate analysis. Moreover, when multivariate analysis was performed with these six covariates and the same endpoint, IL-17 mRNA low expression in peritoneal lavage and tumor size were found to be independent significant predictive factors for prognosis (Table II; HR, 7.91; 95\% CI, 1.65-38.03; $\mathrm{P}=0.0098)$.

Correlation between IL-17-positive cells in primary tumor tissues and IL-17 mRNA expression in peritoneal lavage. To examine the correlation in IL-17 production between the level in peritoneal lavage and in the primary tumor tissues, 


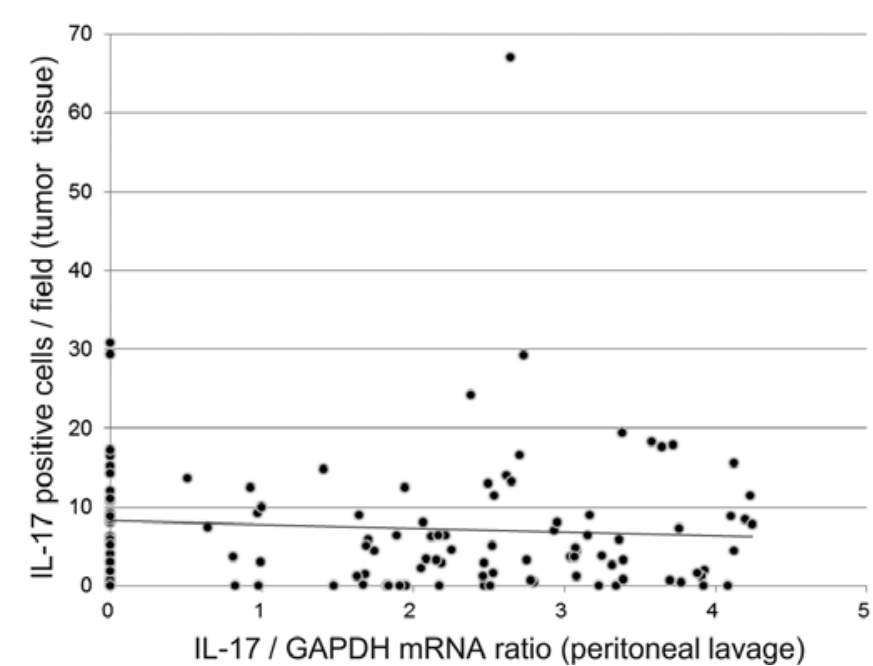

Figure 3. Correlation between IL-17-positive cells in tumor tissues and IL-17 mRNA expression in peritoneal wash. Immunohistochemical staining for IL-17 in primary tumor tissues was performed in the same patient samples whose peritoneal lavage was examined for IL-17 mRNA. The longitudinal axis shows IL-17-positive cells per field in the tumor tissues. The horizontal axis shows the IL-17/GAPDH mRNA ratio in peritoneal lavage.

we performed immunohistochemical staining for IL-17 in the primary tumors in the same patient samples. In the primary tumor tissues, IL-17 immunoreactive cells were detected in the cytoplasm of mononuclear cells; however, none of the tumor cells were stained for IL-17. IL-17-producing cells in the tumor tissues were $7.30 \pm 0.82$ (mean $\pm \mathrm{SE}$ ) per field. There was no correlation between the number of IL-17-positive cells in the tumor tissues and IL-17 mRNA expression in the peritoneal wash ( $\mathrm{r}=0.092 ; \mathrm{P}=0.329)$ (Fig. 3).

Analysis of IL-17-producing cells in peritoneal lavage. Immunohistochemical staining of peritoneal wash revealed that mononuclear cells were stained for IL-17. However, neither tumor cells nor mesothelial cells were stained for IL-17. To identify which mononuclear cells produced IL-17 in the peritoneal lavage, we performed flow cytometric analysis using anti-IL-17, -CD3, -CD4, -CD8 and $-\gamma \delta$ TCR antibodies. CD3 ${ }^{+}$ T cells produced IL-17, while $\gamma \delta$ T cells did not produce IL-17 (Fig. 4A). CD4 ${ }^{+} \mathrm{T}$ cells mainly produced IL-17, and a small population of $\mathrm{CD}^{+} \mathrm{T}$ cells also produced IL-17. The mean percentage of IL-17-positive $\mathrm{CD}^{+} \mathrm{T}$ cells among the total IL-17-positive cell population was only $27.6 \pm 4.85 \%(n=5)$, and in contrast, IL-17-positive $\mathrm{CD}^{+} \mathrm{T}$ cell population was $72.2 \pm 4.86 \%(n=5)$. Representative flow cytometry analysis is shown in Fig. 4B.

\section{Discussion}

In the present study, we demonstrated that in patients who underwent R0 resection, the prognosis of patients in the IL-17 mRNA low expression group was significantly poorer than those in the high expression group. This is the first study evaluating the prognostic value of IL-17 detection by real-time RT-PCR in peritoneal lavage as a valuable prognostic factor in gastric cancer.

IL-17 was originally identified as a proinflammatory cytokine that induces neutrophils, and previous studies also have shown that inflammation is linked to cancer development and progression. It has recently been reported that the levels of IL-17-producing cells are significantly increased in tumor tissues, peripheral blood, malignant ascites fluid, and malignant pleural effusion from a variety of cancer patients (13,14,26-28). Despite recent advances in our understanding of the function of Th17 cells in humans, very little is known about their prevalence and tumor immunosurveillance.

In mice, overexpression of IL-17 by gene transduction into tumor cells promoted tumor growth through angiogenesis (18), but seemingly in contrast, IL-17 also suppressed tumor growth
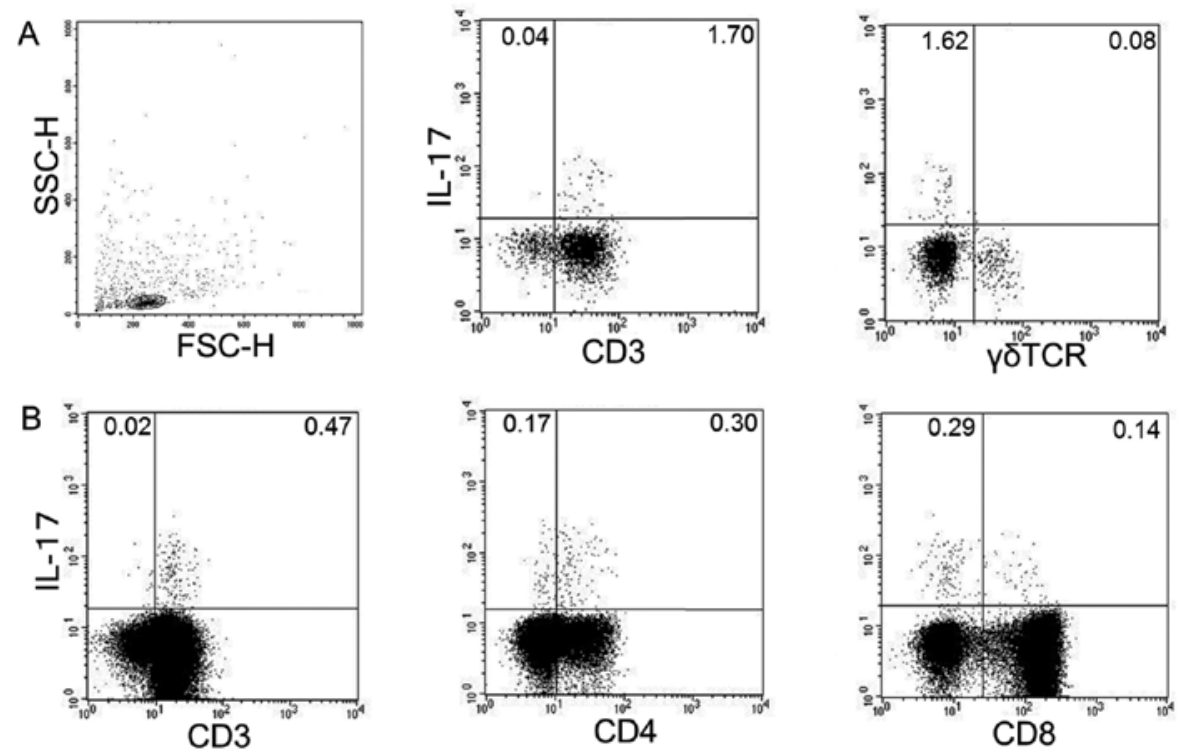

Figure 4. Flow cytometric analysis using anti-IL-17, -CD3, -CD4, -CD8 and $-\gamma \delta$ TCR antibodies to identify IL-17-producing cells in peritoneal washes. (A) Cells were stained with PE-anti-IL-17 mAb, PerCP-anti-CD3 mAb and FITC-anti- $\gamma \delta$ TCR mAb after stimulation with PMA and ionomycin for 5 h. (B) Cells were stained with PE-anti-IL-17 mAb, PerCP-anti-CD3 mAb, FITC-anti-CD4 mAb and FITC-anti-CD8 mAb after stimulation with PMA and ionomycin for $5 \mathrm{~h}$. Representative flow cytometry plots using peritoneal washes from subjects with cytology class II. 
via a T-cell-dependent mechanism (19). Whether IL-17 promotes tumor growth or regulates antitumor responses remains controversial.

In humans, there are several reports concerning intratumoral expression of IL-17 and its prognostic role in several cancer types such as hepatocellular carcinoma (HCC), colon, esophageal and gastric cancer. In HCC, colon, and prostate cancer patients, intratumoral IL-17-positive cells were found to be correlated with poor survival $(17,29,30)$. Our previous study showed that Th17 cells infiltrated the tumor and secreted IL-17 in the tumor microenvironment, leading to tumor progression through angiogenesis and neutrophil infiltration in patients with gastric cancer. In the present study, we hypothesized that IL-17 promotes tumor progression in the peritoneal cavity, based on our previous study suggesting that IL-17 is related to tumor progression in the tumor microenvironment. We quantitatively analyzed the expression levels of IL-17 mRNA in peritoneal lavage from gastric cancer patients. Based on the survival curves, among the 79 patients who underwent $\mathrm{R} 0$ resection, the patients in the IL-17 mRNA low expression group had a significantly poorer prognosis than the patients in the IL-17 mRNA high expression group. This result was contradictory to our hypothesis. This discrepancy may be explained by the difference in the impact of IL-17 on tumor progression in the thoracoabdominal cavity and in tumor tissue. In fact, in the present study, there were no correlations noted between primary tumor tissues and peritoneal wash in terms of IL-17 expression. In lung cancer, Ye et al (26) reported that patients with a higher proportion of Th17 cells in malignant pleural effusion exhibited significantly longer overall survival than patients with a lower proportion of Th17 cells. Similarly, in ovarian cancer, the expression of IL-17 in ascites was analyzed, and patients with a higher IL-17 expression in ascites had a significantly lower death hazard than those with a lower IL-17 expression (28).

Most recently, it has been reported that $\mathrm{CD}^{+} \mathrm{T}$ cells that produce IL-17 ( Tc17 cells) are abundant in gastric cancer tissue, and the percentage of Th17 cells is relatively lower than that of Tc17 cells in tumors. The intratumoral Tc17 cell percentage was significantly associated with tumor progression and poor prognosis (31). In the present study, flow cytometric analysis showed that $\mathrm{CD}^{+}$Th17 cells predominantly produced IL-17 in the peritoneal lavage; however, the percentage of Tc17 cells was lower than that of Th17. This suggests that IL-17producing $\mathrm{T}$ cells are different between tumor tissue and the abdominal cavity, and the potential role of IL-17 could also be different in the tumor microenvironment between tumor tissue and the abdominal cavity.

There is another reason why the results of the present study were in conflict with our expectations. This may be because the role of IL-17 is different before and after the tumor is established. In the present study, the expression levels of IL-17 were significantly higher in peritoneal carcinomatosis-positive cases than those of negative cases, while they were not associated with cytologic examination (Table IA). Once tumor cells attach to the peritoneum, IL-17 may play a role as a tumor growth cytokine through angiogenesis to a greater exent than its role in regulating antitumor responses. Our results suggest that endogenous IL-17 plays different roles before tumor attachment versus in established tumor growth. Furthermore, in the abdominal cavity, previous studies indicate that peritoneal mesothelial cells secrete various cytokines and growth factors, such as IL-6, IL-8, IL-1 $\alpha$ and $\beta$, granulocyte colony stimulating factor (G-CSF), as well as vascular endothelial growth factor (VEGF) and fibroblast growth factor (FGF)-2. These results indicate that peritoneal mesothelial cells are one of the central elements of the cytokine network controlling disease processes in the abdominal cavity. Kryczek et al suggested that, in the peritoneal cavity, IL-17 is positively associated with INF- $\gamma$ effector T cells and Th1-type chemokines, CXCL9 and CXCL10, but not with Th2-type chemokines, CXCL12 and CCL22, in ovarian cancer ascites. Mechanistically, Th17 cellderived IL-17 and INF- $\gamma$ were found to synergistically induce the production of CXCL9 and CXCL10 and in turn promote effector T-cell migration (28). Thus, IL-17 may function as a polyfunctional cytokine profile in human tumors. There is no doubt that the role of IL-17 is highly complicated, and it remains controversial whether IL-17 promotes tumor growth or regulates the antitumor response.

In conclusion, IL-17 mRNA expression in peritoneal lavage detected by real-time RT-PCR is a reliable prognostic factor for patients with curative resection in gastric cancer. Low IL-17 gene expression in the peritoneal cavity may correlate with cancer development in the peritoneal cavity and poor prognosis in patients with gastric cancer.

\section{References}

1. Takahashi I, Matsusaka T, Onohara T, et al: Clinicopathological features of long-term survivors of scirrhous gastric cancer. Hepatogastroenterology 47: 1485-1488, 2000.

2. Ito S, Nakanishi H, Kodera Y, Mochizuki Y, Tatemastu M and Yamamura Y: Prospective validation of quantitative CEA mRNA detection in peritoneal washes in gastric carcinoma patients. $\mathrm{Br} \mathbf{J}$ Cancer 93: 986-992, 2005.

3. Bonenkamp JJ, Songun I, Hermans J and van de Velde CJ: Prognostic value of positive cytology findings from abdominal washings in patients with gastric cancer. Br J Surg 83: 672-674, 1996.

4. Kodera Y, Nakanishi H, Yamamura Y, et al: Prognostic value and clinical implications of disseminated cancer cells in the peritoneal cavity detected by reverse transcriptase-polymerase chain reaction and cytology. Int J Cancer 79: 429-433, 1998.

5. Fujii S, Kitayama J, Kaisaki S, et al: Carcinoembryonic antigen mRNA in abdominal cavity as a useful predictor of peritoneal recurrence of gastric cancer with serosal exposure. J Exp Clin Cancer Res 21: 547-553, 2002.

6. Tokuda K, Natsugoe S, Nakajo A, et al: Clinical significance of CEA-mRNA expression in peritoneal lavage fluid from patients with gastric cancer. Int J Mol Med 11: 79-84, 2003.

7. Boku T, Nakane Y, Minoura T, et al: Prognostic significance of serosal invasion and free intraperitoneal cancer cells in gastric cancer. Br J Surg 77: 436-439, 1990.

8. Kodera Y, Nakanishi H, Ito S, et al: Quantitative detection of disseminated free cancer cells in peritoneal washes with real-time reverse transcriptase-polymerase chain reaction: a sensitive predictor of outcome for patients with gastric carcinoma. Ann Surg 235: 499-506, 2002.

9. Oyama K, Terashima M, Takagane A, Maesawa C, et al: Prognostic significance of peritoneal minimal residual disease in gastric cancer detected by reverse transcription-polymerase chain reaction. Br J Surg 91: 435-443, 2004.

10. Zeimet AG, Widschwendter M, Knabbe C, et al: Ascitic interleukin-12 is an independent prognostic factor in ovarian cancer. J Clin Oncol 16: 1861-1868, 1998.

11. Majima T, Ichikura T, Seki S, Takayama E, Hiraide H and Mochizuki H: Interleukin-10 and interferon-gamma levels within the peritoneal cavity of patients with gastric cancer. J Surg Oncol 78: 124-130, 2001. 
12. Zhang B, Rong G, Wei H, et al: The prevalence of Th17 cells in patients with gastric cancer. Biochem Biophys Res Commun 374: $533-537,2008$

13. Maruyama T, Kono K, Mizukami Y, et al: Distribution of Th17 cells and FoxP3(+) regulatory $\mathrm{T}$ cells in tumor-infiltrating lymphocytes, tumor-draining lymph nodes and peripheral blood lymphocytes in patients with gastric cancer. Cancer Sci 101: $1947-1954,2010$

14. Miyahara Y, Odunsi K, Chen W, Peng G, Matsuzaki J and Wang RF: Generation and regulation of human CD4+ IL-17producing $\mathrm{T}$ cells in ovarian cancer. Proc Natl Acad Sci USA 105: 15505-15510, 2008.

15. Langowski JL, Zhang X, Wu L, et al: IL-23 promotes tumour incidence and growth. Nature 442: 461-465, 2006.

16. Kato T, Furumoto H, Ogura T, et al: Expression of IL-17 mRNA in ovarian cancer. Biochem Biophys Res Commun 282: 735-738, 2001.

17. Sfanos KS, Bruno TC, Maris CH, et al: Phenotypic analysis of prostate-infiltrating lymphocytes reveals $\mathrm{T}_{\mathrm{H}} 17$ and $\mathrm{T}_{\text {reg }}$ skewing. Clin Cancer Res 14: 3254-3261, 2008.

18. Numasaki M, Fukushi J, Ono M, et al: Interleukin-17 promotes angiogenesis and tumor growth. Blood 101: 2620-2627, 2003.

19. Benchetrit F, Ciree A, Vives V, et al: Interleukin-17 inhibits tumor cell growth by means of a T-cell-dependent mechanism. Blood 99: 2114-2121, 2002

20. Zou W and Restifo NP: T(H)17 cells in tumour immunity and immunotherapy. Nat Rev Immunol 10: 248-256, 2010.

21. Iida T, Iwahashi M, Katsuda M, et al: Tumor-infiltrating CD4 ${ }^{+}$ Th17 cells produce IL-17 in tumor microenvironment and promote tumor progression in human gastric cancer. Oncol Rep 25: 1271-1277, 2011.

22. Kryczek I, Wei S, Zou L, et al: Cutting edge: Th17 and regulatory $\mathrm{T}$ cell dynamics and the regulation by IL-2 in the tumor microenvironment. J Immunol 178: 6730-6733, 2007.
23. Lissoni $\mathrm{P}$, Mandalà M, Curigliano G, et al: Progress report on the palliative therapy of 100 patients with neoplastic effusions by intracavitary low-dose interleukin-2. Oncology 60: 308-312, 2001.

24. Lenzi R, Rosenblum M, Verschraegen C, et al: Phase I study of intraperitoneal recombinant human interleukin 12 in patients with Müllerian carcinoma, gastrointestinal primary malignancies, and mesothelioma. Clin Cancer Res 8: 3686-3695, 2002.

25. Fu QG, Meng FD, Shen XD and Guo RX: Efficacy of intraperitoneal thermochemotherapy and immunotherapy in intraperitoneal recurrence after gastrointestinal cancer resection. World J Gastroenterol 8: 1019-1022, 2002.

26. Ye ZJ, Zhou Q, Gu YY, et al: Generation and differentiation of IL-17-producing $\mathrm{CD}^{+} \mathrm{T}$ cells in malignant pleural effusion. $\mathrm{J}$ Immunol 185: 6348-6354, 2010.

27. Derhovanessian E, Adams V, Hähnel K, et al: Pretreatment frequency of circulating IL- $17^{+} \mathrm{CD} 4^{+} \mathrm{T}$-cells, but not Tregs, correlates with clinical response to whole-cell vaccination in prostate cancer patients. Int J Cancer 125: 1372-1379, 2009.

28. Kryczek I, Banerjee M, Cheng P, et al: Phenotype, distribution, generation, and functional and clinical relevance of Th17 cells in the human tumor environments. Blood 114: 1141-1149, 2009.

29. Zhang JP, Yan J, Xu J, et al: Increased intratumoral IL-17producing cells correlate with poor survival in hepatocellular carcinoma patients. J Hepatol 50: 980-989, 2009.

30. Liu J, Duan Y, Cheng X, et al: IL-17 is associated with poor prognosis and promotes angiogenesis via stimulating VEGF production of cancer cells in colorectal carcinoma. Biochem Biophys Res Commun 407: 348-354, 2011.

31. Zhuang Y, Peng LS, Zhao YL, Shi Y, Mao XH, et al: $\mathrm{CD} 8^{+} \mathrm{T}$ cells that produce interleukin-17 regulate myeloid-derived suppressor cells and are associated with survival time of patients with gastric cancer. Gastroenterology 143: 951.e8-962.e8, 2012. 